\title{
A CROSS-SECTIONAL STUDY TO EVALUATE THE OUTCOME OF PATIENTS ADMITTED TO PAEDIATRIC INTENSIVE CARE UNIT BY USING PAEDIATRIC MULTIPLE ORGAN DYSFUNCTION SCORE (P-MODS) AT KLES TERTIARY CARE HOSPITAL AND MRC, BELGAUM
}

\author{
Mousin Mustafa Batt ${ }^{1}$ Roopa M. Bellad ${ }^{2}$ \\ ${ }_{1}$ Postgraduate Student, Department of Paediatrics, Jawaharlal Nehru Medical College, Belgaum, Karnataka. \\ 2 Professor and HOD, Department of Paediatrics, Jawaharlal Nehru Medical College, Belgaum, Karnataka.
}

\section{ABSTRACT}

\section{BACKGROUND}

Objectives- Mortality in PICU is related to the number of organ dysfunction. The PMOD score as a continuous variable, which defines and predicts the number of organ dysfunction in successive day of ICU stay can be a good alternative to other scoring systems such as PIM and PRISM use as a surrogate for prediction of mortality in PICU.

The present study was planned in an attempt to evaluate the outcome of patients admitted to PICU by using Paediatric Multiple Organ Dysfunction Score (P-MODS).

\section{MATERIALS AND METHODS}

This one-year cross-sectional study was done in the PICU under the Department of Paediatrics, KLES Dr. Prabhakar Kore Hospital and Medical Research Centre, Belgaum from January 2014 to December 2014. During the study period, there were a total of 694 admissions in PICU of which eligible 100 were studied.

\section{RESULTS}

Of the 100 children studied, 59\% of the children were boys with boy to girl ratio of 1.43:1 and more than one-third of the children (37\%) were aged $<1$ year and the mean age was $4.65 \pm 4.60$ years. $56 \%$ of the children were from Class II socioeconomic status according to modified B. G. Prasad's classification. The mortality rate was $18 \%$. MODS was noted in $74 \%$ of the children and mortality was significantly associated with MODS $(\mathrm{p}=0.001)$. Ventilatory support was noted in $24 \%$ of the children. In majority of the children $(70 \%)$, the length of hospital stay was $\leq 3$ days. Most of the children (42\%) had P-MOD score of 4 to 7 . The mean PMOD scores among the non-survivors were significantly high compared to non-survivors $(\mathrm{p}<0.001)$. The percentage of death in children with 0 to 3 P-MOD scores was $3.3 \%$, which was high reaching $60 \%$ among the children with P-MOD scores of $\geq 12$. The mean PMOD scores increased significantly with increase in organ involvement $(\mathrm{p}<0.001)$. Using the cut-off point of 7.5, PMOD score showed higher sensitivity (77.78\%) and specificity (82.93\%) in predicting outcome.

\section{CONCLUSION}

PMOD score strongly correlates with the PICU mortality and no. of organ dysfunction and can be used in PICU for the prediction of outcome.

\section{KEYWORDS}

Multiple Organ Dysfunction Syndrome; Paediatric Intensive Care Unit; Paediatric Organ Dysfunction Score.

HOW TO CITE THIS ARTICLE: Batt MM, Bellad RM. A cross-sectional study to evaluate the outcome of patients admitted to paediatric intensive care unit by using paediatric multiple organ dysfunction score (p-mods) at KLES tertiary care hospital and MRC, Belgaum. J. Evolution Med. Dent. Sci. 2017;6(45):3542-3547, DOI: 10.14260/Jemds/2017/763

\section{BACKGROUND}

Paediatric Intensive Care Units (PICU) aim at promoting qualified care with the objective of achieving the best results and better progress for critically ill children. ${ }^{1}$ The rapid technology advances in PICU over the past decades has resulted in the most sophisticated care of the sick children, thus making these units prepared to treat cases of high complexity.2,3

Critical care technological advances coupled with spiralling cost of the medical care has resulted in need for outcome analysis including risk prediction. ${ }^{2,3}$

Financial or Other, Competing Interest: None.

Submission 03-04-2017, Peer Review 24-05-2017,

Acceptance 31-05-2017, Published 05-06-2017.

Corresponding Author:

Dr. Mousin Mustafa Batt,

Postgraduate Student,

Department of Paediatrics, Jawaharlal Nehru Medical College,

Nehru Nagar, Belgaum-590010, Karnataka, India.

E-mail: mohsin.azad28@gmail.com

DOI: $10.14260 /$ jemds $/ 2017 / 763$

(c) $(\rightarrow)$
Thus, it is necessary to characterise the disease severity, assessing its prognosis. In the context of intensive care management, a rational and objective way to define and quantify severity of illness is through the development of probability models predicting mortality risk. ${ }^{4,5}$ Such models allow an increased understanding of the effectiveness of different medical interventions, development of standards that may guide health care providers in optimising the use of available medical resources. Moreover, the use of this information may aid in the decision making process by physicians and parents. ${ }^{4}$ Since they compare mortality adjusted by disease severity, these scores can also be used for comparisons between clinical trials and for planning technological resources in this area. $1,6,7$

Mortality and length of hospital stay are examples of the most used outcomes. Scoring systems are arrived at evaluation of the patient's mortality risk in the ICU by assigning a score to patient and predicting the outcome. However, patient's mortality is not only affected by ICU performance, but also depends on many other factors such as demographic and clinical characteristic of population, 
infrastructure and non-medical factors (administrator, management and organisation), case mix and admission practice. ${ }^{8}$ Therefore, there is need for field testing of these scoring system in setting different from the one in which they were originally developed.

The principal scores that have been developed for the paediatric population are the PRISM (Paediatric Risk of Mortality) $^{9}$ and PIM (Paediatric Index of Mortality) ${ }^{10}$ with their most recent versions being PRISM III and PIM-2.11 These scores were developed by identifying variables relevant to mortality risk and scoring them after a multivariate statistical analysis by logistic regression. ${ }^{12}$

As mortality is tightly linked to Multiple Organ Dysfunction Syndrome (MODS) and prevalence of MODS in PICUs range from $11 \%$ to $18 \%$, the MODS scores constructed as a continuous variable may be a good alternative outcome to use as a surrogate for death in the PICU. These scores may allow the evaluation of the efficacy of ICU therapies when survival is used as the primary outcome. ${ }^{13}$

Several MODS scoring systems have been developed and validated mainly in adults. ${ }^{13}$ However, an equivalent MODS scoring system is not available for critically ill children. The ideal probability model/scoring system would be institution independent and population independent. Hence, the present study was planned in an attempt to evaluate the outcome of patients admitted to PICU by using Paediatric Multiple Organ Dysfunction Score (P-MODS) in our setting.

\section{MATERIALS AND METHODS}

The present one-year prospective study was conducted from period from January 2014 to December 2014. Patients admitted in Paediatric Intensive Care Unit of the Department of Paediatrics, teaching hospital attached to KLE University's JN Medical College, Belgaum. The study included 100 children who fulfilled the selection criteria. Based on the consecutive sampling, every consecutive child admitted to PICU was enrolled in the study (Figure 1).

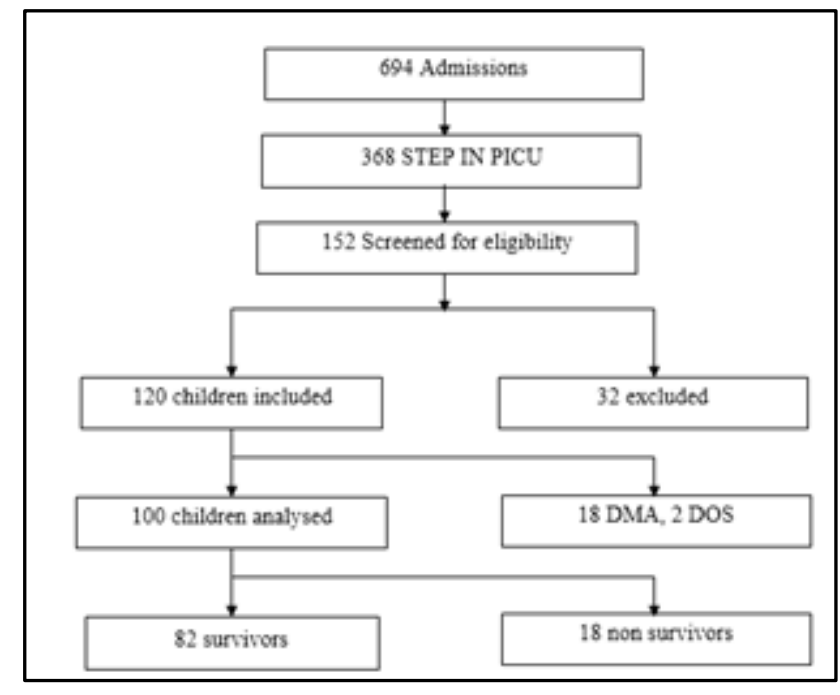

Figure 1. Consort Diagram showing the Study Population

Children younger than 18 years, both medical and surgical cases and willing to participate in the study were enrolled. The children less than one month old, congenital heart diseases, staying in PICU $<1$ hour, history of burns and discharged against medical advice were excluded from the study. Prior to the commencement, the ethical clearance was obtained from the Ethical and Research Committee, Jawaharlal Nehru Medical College, Belgaum. All the parents/caregivers of the infants fulfilling selection criteria were explained about the purpose of the study and a written informed consent was obtained from parents/caregivers to participate in the study before enrolment.

At admission, a detailed history and systemic examination was performed to assess the vital parameters by the paediatrician and the findings were recorded on a predesigned and pretested proforma. The sociodemographic data obtained from the parents was also recorded. After one hour of admission, children were evaluated by the resident paediatrician and every consecutive child admitted in the PICU was enrolled in the study till the sample was achieved during the study period. The investigations including lactic acid, total bilirubin, fibrinogen, blood urea nitrogen and $\mathrm{PaO}_{2} / \mathrm{FIO}_{2}$ ratio were done. These investigations were repeated at an interval of 24 hours till discharge and noted in predesigned and pretested proforma.

The admission PMOD scores were calculated from the data recorded at admission and daily scores from the data recorded. The calculation of PMOD score was based on highest values within the observed range. The individual influence of each variable was summed to produce a final PMOD scores. The grading scale for MODS was set from 0 to 4 for each variable, where 0 represented none or minimal dysfunction, whereas a grade of 4 represented severe dysfunction (Table 1).

Based on the clinical presentation, physical examination findings, laboratory investigations and diagnosis, patients were evaluated for the number organ failure, involvement of system at admission, mode of management (Routine care like IV fluid, antibiotics, oxygen supplement, inotropes, requirement of blood transfusion, immunoglobulins and special care like ventilatory support and dialysis) were assessed. The study end point was outcome (survival and non-survival) and length of hospital stay (number of days from admission to discharge).

\section{Statistical Analysis}

The data obtained was coded and entered into Microsoft Excel spreadsheet and data was analysed using SPSS version 20. The categorical data was expressed in terms of rates, ratios and percentages and the continuous data was expressed in terms of mean \pm standard deviation. The association between the outcome, clinical and demographic characteristics was tested using Chi-square test or Fisher's exact test. The discrimination between survivors and nonsurvivors was made using the receiver operating characteristic curve (ROC curve). ${ }^{14}$ If the AUC (Area under Curve) is 0.9 or more, it is considered excellent discrimination; $0.80-0.89$ is considered good; and $0.70-0.79$ is considered as fair. The accuracy of PMOD scores in discriminating the survival was expressed in terms of sensitivity, specificity, positive predictive value, negative predictive value and positive likelihood ratio. The prediction of probability of death was determined by goodness of fit predictive model, logistic regression analysis and odds ratio. The association in terms of odds ratio was assessed by Hosmer-Lemeshow summary Chi-square test and also by the 
ROC analysis. The correlation of PMOD scores with organ failure and hospital stay was done using Pearson's correlation co-efficient. At $95 \%$ confidence interval, a probability (p) value of $\leq 0.050$ was considered as statistically significant.

\begin{tabular}{|c|c|c|}
\hline Variables & Range & Score \\
\hline \multirow[t]{5}{*}{ Lactic acid (mmol/L) } & $<1$ & 0 \\
\hline & $1-2$ & 1 \\
\hline & $2-5$ & 2 \\
\hline & $5-7.5$ & 3 \\
\hline & $>7.5$ & 4 \\
\hline \multirow[t]{5}{*}{$\mathrm{PaO} 2 / \mathrm{FiO} 2(\mu \mathrm{mol} / \mathrm{L})$} & $<8.5$ & 0 \\
\hline & $8.50-34.2$ & 1 \\
\hline & $34.2-85.5$ & 2 \\
\hline & $85.5-171$ & 3 \\
\hline & $>171$ & 4 \\
\hline \multirow[t]{5}{*}{ Fibrinogen $(\mu \mathrm{mol} / \mathrm{L})$} & $>4.4$ & 0 \\
\hline & $4.4-3.7$ & 1 \\
\hline & $3.7-3.0$ & 2 \\
\hline & $3.0-2.0$ & 3 \\
\hline & $<2.0$ & 4 \\
\hline \multirow[t]{5}{*}{ Bilirubin (mg/dL) } & $<0.5$ & 0 \\
\hline & $0.5-2.0$ & 1 \\
\hline & $2.0-5.0$ & 2 \\
\hline & $5.0-10.0$ & 3 \\
\hline & $>10.0$ & 4 \\
\hline Blood urea nitrogen & $<20$ & 0 \\
\hline \multirow[t]{4}{*}{$(\mathrm{mg} / \mathrm{dL})$} & $20-40$ & 1 \\
\hline & $41-60$ & 2 \\
\hline & $61-80$ & 3 \\
\hline & $>80$ & 4 \\
\hline
\end{tabular}

Table 1. Paediatric Multiple Organ Dysfunction (P-MODS) Score developed by Graciano AL et al ${ }^{15}$

\section{RESULTS}

Male constituted 59\% and $41 \%$ were females with male-tofemale ratio of 1.43:1. Majority of the children improved (82\%) (Graph 1). No association was found between gender and outcome $(\mathrm{p}=0.743)$. More than one-third of the children $(37 \%)$ were aged $<1$ year and the mean age was $4.65 \pm 4.60$ years. However, the outcome was comparable in different age groups $(\mathrm{p}=0.325)$. Class II socioeconomic status was noted in $56 \%$ of the children according to modified BG Prasad's classification. No association was noted between socioeconomic class and outcome $(\mathrm{p}=1.000)$.

Cough, cold and fever (77\%), loose stool and vomiting (29\%), petechiae and ecchymosis (28\%), and confusion (22\%) were the commonest clinical features at presentation. Majority of the children had involvement of CVS system $(82.3 \%)$. MODS-2 or more organ was noted in $74 \%$ of the children and mortality was significantly associated with MODS $(p=0.001)$. Mortality was also observed to be high in isolated organ failure (i.e. respiratory $p=0.002$ and hepatic system $p=0.040$ ). Wide variation was observed in the diagnosis of the children and most of the children were diagnosed to have dengue fever $(18 \%)$ followed by febrile seizures (4\%).

Ventilatory support was indicated in $24 \%$ of the children. Significantly higher number of non-survivors required ventilatory support $(\mathrm{p}<0.001)$. In majority of the children (70\%), the length of hospital stay was $\leq 3$ days and mean length of hospital stay was $2.81 \pm 1.82$ days. However, mean length of hospital stay was comparable in survivors and nonsurvivors $(p=0.485)$ and it did not differ significantly with organ failures (two organs, $\mathrm{p}=0.845$ ) and (three organs, $\mathrm{p}=$ 0.070).

Most of the children (42\%) had P-MOD score of 4 to 7 . The mean PMOD scores among the non-survivors were significantly high compared to those who survived $(\mathrm{p}<$ 0.001). The survival rate in children with P-MOD scores ranging from 0 to 3 and 4 to 7 was $96 \%$ and $92.9 \%$. The percentage of death in children with 0 to 3 P-MOD scores, i.e. $3.3 \%$ which gradually increased with increase in P-MOD scores reaching $60 \%$ among the children with P-MOD scores of $\geq 12$ (Table 1). The mean PMOD scores increased significantly with increase in organ involvement $(\mathrm{p}<0.001)$ (Table 2). Using the cut-off point of 7.5 PMOD score showed higher sensitivity (77.78\%) and specificity $(82.93 \%)$ in predicting outcome (50\% PPV and $94.44 \%$ NPV; positive likelihood ratio 4.56 and negative likelihood ratio 0.27 ) (Table 5).

The ROC curve for predicting outcome using PMOD scores yielded maximum sensitivity and specificity at cut-off point of 7.5. (If the AUC is 0.9 or more it is considered excellent discrimination, $0.80-0.89$ is considered good and $0.70-0.79$ is considered as fair) (Graph 2).

Prediction of probability of death in PICU according to PMOD score showed that a child with P-MOD score of 0 had a $1.2 \%$ chance of dying in the PICU, whereas a score of 11 predicted $51 \%$ chance of death (Table 4 ). This means that the probability of death increased with P-MOD scores. Logistic regression analysis showed odds of children dying increases by 3.118 unit increase in P-MOD score (OR 22.591; 95\% CI 0.56 to 0.83 ; $\mathrm{p}<0.001$ ), i.e. for an increase of 1 in P-MOD score, child's odds of death increases by $22 \%$. The best indicators of poor outcome in our study were organ dysfunction, requirement of ventilatory support and PMOD score.
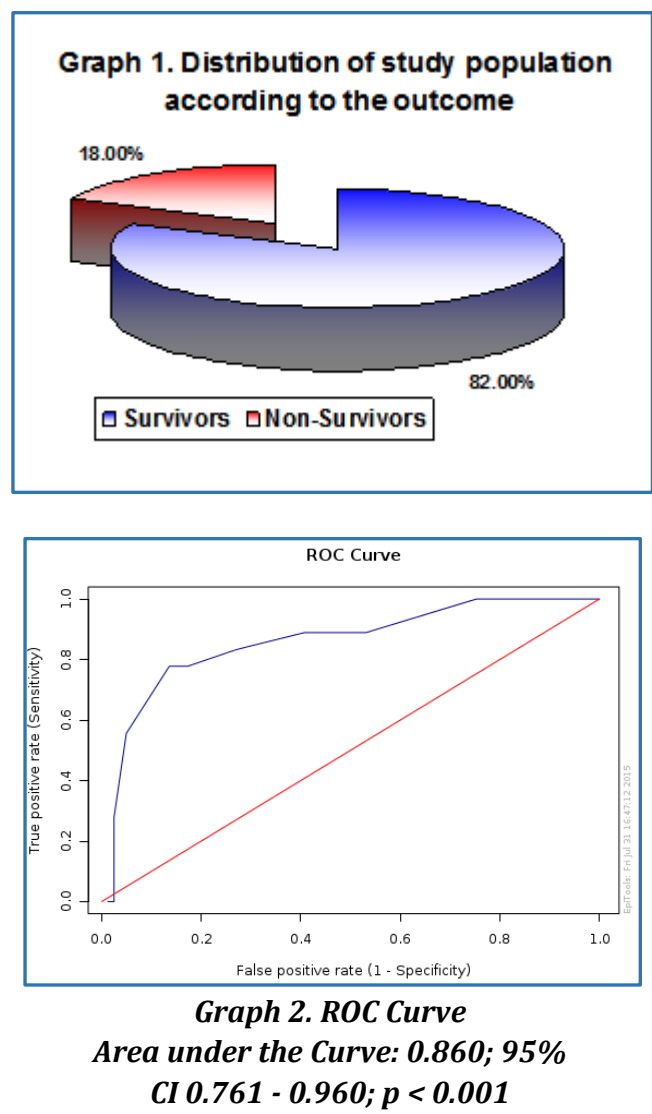


\begin{tabular}{|c|c|c|c|c|c|}
\hline \multirow{3}{*}{$\begin{array}{l}\text { PMOD } \\
\text { Scores }\end{array}$} & \multirow{3}{*}{$\begin{array}{c}\text { Total } \\
\text { Number }\end{array}$} & \multicolumn{4}{|c|}{ Outcome } \\
\hline & & \multicolumn{2}{|c|}{ Survivors } & \multicolumn{2}{|c|}{ Non-Survivors } \\
\hline & & No & $\%$ & No & $\%$ \\
\hline $0-3$ & 30 & 29 & 96.7 & 1 & 3.3 \\
\hline $4-7$ & 42 & 39 & 92.9 & 3 & 7.1 \\
\hline $8-11$ & 23 & 12 & 52.2 & 11 & 47.8 \\
\hline$\geq 12$ & 5 & 2 & 40.0 & 3 & 60.0 \\
\hline \multicolumn{6}{|c|}{ Table 2. Association of P-MOD Score with Outcome } \\
\hline
\end{tabular}

$\mathrm{P}<0.001$

\begin{tabular}{|c|c|c|c|c|c|c|c|c|}
\hline \multirow{2}{*}{$\begin{array}{c}\text { P-MOD } \\
\text { Score }\end{array}$} & \multicolumn{8}{|c|}{ Organ Dysfunction } \\
\cline { 2 - 9 } & \multicolumn{2}{|c|}{ One } & \multicolumn{2}{c|}{ Two } & \multicolumn{2}{c|}{ Three } & \multicolumn{2}{c|}{ Four } \\
\cline { 2 - 9 } & No. & $\%$ & No. & $\%$ & No. & $\%$ & No. & $\%$ \\
\hline 3 or less & 15 & 75.00 & 4 & 20.00 & 0 & 0.00 & 1 & 5.00 \\
\hline 4 to 7 & 8 & 19.05 & 28 & 66.67 & 5 & 11.90 & 1 & 2.38 \\
\hline 8 to 11 & 0 & 0.00 & 13 & 56.52 & 10 & 43.48 & 0 & 0.00 \\
\hline$>12$ & 3 & 20.00 & 8 & 53.33 & 4 & 26.67 & 0 & 0.00 \\
\hline Total & $\mathbf{2 6}$ & $\mathbf{2 6 . 0 0}$ & $\mathbf{5 3}$ & $\mathbf{5 3 . 0 0}$ & $\mathbf{1 9}$ & $\mathbf{1 9 . 0 0}$ & $\mathbf{2}$ & $\mathbf{2 . 0 0}$ \\
\hline Table 3. Association of PMOD Score with Organ \\
Dysfunction \\
\hline
\end{tabular}

$\mathrm{P}<0.001$

\begin{tabular}{|c|c|c|c|c|c|c|c|c|}
\hline \multirow{2}{*}{$\begin{array}{c}\text { PMOD } \\
\text { Scores }\end{array}$} & \multicolumn{9}{|c|}{ Length of Hospital Stay } & \multirow{2}{*}{ Total } \\
\cline { 2 - 9 } & $\mathbf{3}$ or less & \multicolumn{2}{|c|}{$\mathbf{4}$ to 7} & $\mathbf{8}$ to 12 & \multicolumn{1}{|c|}{} \\
\cline { 2 - 9 } & $\mathbf{N o}$ & $\mathbf{\%}$ & $\mathbf{N o}$ & $\mathbf{\%}$ & No & $\mathbf{\%}$ & No & $\%$ \\
\hline $0-3$ & 18 & 60.00 & 11 & 36.67 & 1 & 3.33 & 30 & 100.00 \\
\hline $4-7$ & 29 & 69.05 & 13 & 30.95 & 0 & 0.00 & 42 & 100.00 \\
\hline $8-11$ & 18 & 78.26 & 4 & 17.39 & 1 & 4.35 & 23 & 100.00 \\
\hline$\geq 12$ & 5 & 100.00 & 0 & 0.00 & 0 & 0.00 & 5 & 100.00 \\
\hline Total & $\mathbf{7 0}$ & $\mathbf{7 0 . 0 0}$ & $\mathbf{2 8}$ & $\mathbf{2 8 . 0 0}$ & $\mathbf{2}$ & $\mathbf{2 . 0 0}$ & $\mathbf{1 0 0}$ & $\mathbf{1 0 0 . 0 0}$ \\
\hline Table 4. Comparison of PMOD Scores with Length of \\
Hospital Stay \\
\hline
\end{tabular}

$P=0.275$

\begin{tabular}{|c|c|}
\hline P-MOD Score & Probability of Death (\%) \\
\hline 0 & $1.2 \%$ \\
\hline 4 & $7 \%$ \\
\hline 5 & $10 \%$ \\
\hline 8 & $25 \%$ \\
\hline 9 & $33 \%$ \\
\hline 10 & $42 \%$ \\
\hline 11 & $51 \%$ \\
\hline 14 & $77 \%$ \\
\hline 16 & $88 \%$ \\
\hline 18 & $94 \%$ \\
\hline \multicolumn{2}{|c|}{ Table 5. Prediction of Probability of Death in ICU } \\
according to P-MOD Score \\
\hline
\end{tabular}

\begin{tabular}{|c|c|c|c|c|c|c|}
\hline \multirow{2}{*}{$\begin{array}{c}\text { PMOD } \\
\text { Scores }\end{array}$} & \multicolumn{4}{|c|}{ Outcome } & \multicolumn{2}{|c|}{ Total } \\
\cline { 2 - 7 } & Non-Survivors & \multicolumn{2}{|c|}{ Survivors } & \multicolumn{2}{|c|}{} \\
\cline { 2 - 7 } & No. & $\mathbf{\%}$ & No. & $\%$ & No. & $\%$ \\
\hline$\geq 7.5$ & 14 & 50.00 & 14 & 50.00 & 28 & 100.00 \\
\hline$<7.5$ & 4 & 5.56 & 68 & 94.44 & 72 & 100.00 \\
\hline Total & $\mathbf{1 8}$ & $\mathbf{1 8 . 0 0}$ & $\mathbf{8 2}$ & $\mathbf{8 2 . 0 0}$ & $\mathbf{1 0 0}$ & $\mathbf{1 0 0 . 0 0}$ \\
\hline \multicolumn{6}{|c|}{ Table 6. Accuracy of PMOD Scores in Predicting Outcome } \\
\hline
\end{tabular}

$\mathrm{P}<0.001$

\section{DISCUSSION}

In the present study, demographic characteristics like gender $(\mathrm{p}=0.743)$, age $(\mathrm{p}=0.325)$ and socioeconomic status $(\mathrm{p}=$ 0.325 ) did not show any significant correlation with the outcome. Male-to-female ratio in our study was 1.43:1 with $59 \%$ boys and $41 \%$ girls. Similar observations of the gender distribution were reported by studies from developed and developing countries. ${ }^{15,16}$ In a recent study by India conducted to evaluate the usefulness of PIM II reports Male: Female 1.1:1.16 These observations were in agreement with other reports in India. ${ }^{16}$ This difference is common in countries such as India, which have a preference for male gender. The gender distribution pattern of the present study was comparable with the study by Graciano AL et al,15 who reported $56.8 \%$ males and $42.70 \%$ females.

The mortality rate observed in the study was $18 \%$, that is similar to earlier in-house study ${ }^{17}$ of $16.7 \%$ and other studies from developed and developing countries.17,18,19 Contradictory observations with lower mortality rates are reported by the recent studies from India and Hong Kong ranging from $2.1 \%$ to $6.7 \% .^{20,21,19}$ The difference observed in the mortality rate in different studies ${ }^{20,21,19}$ can be explained by the varied inclusion criteria and variable disease patterns and the severity of illness.

The mean PMODS score were significantly high among survivors as compared to survivors $(9.2 \pm 2.75$ vs $4.9 \pm 3.28$; $\mathrm{p}<0.001)$. Studies evaluating other scoring systems for predicting the outcome in PICU also have reported increase in the predictive scores among the non-survivors. ${ }^{1,20}$

Majority of children (42\%) had P-MOD score of 4 to 7 followed by $\leq 3(30 \%)$ and 8 to $11(23 \%)$. A significant association of the PMOD score with mortality was observed in the study $(\mathrm{p}<0.001)$. Majority of children $(42 \%)$ had PMOD score of 4 to 7 followed by $\leq 3$ (30\%). The survival rate was high in children with P-MOD scores of 0 to 3 and 4 to 7 ( $96 \%$ and $92.9 \%$, respectively). The percentage of death with PMOD score 0 - 3 was low $3.3 \%$ and gradually increased with higher PMOD scores $(60 \%$ in children with P-MOD scores of $\geq$ 12). These findings of the present study were similar to the study developed to validate PMOD score as an outcome measure by Graciano AL et al. ${ }^{15}$ This observation demonstrates that with the increase in PMOD score, there is increase in mortality. The association of increase in mortality with increase in the predictive score in the intensive care has been also reported by other studies both in developed and developing countries.

In this study PMOD, scores were significantly high in children with 3 organ dysfunction $54.55 \%$ and 2 organ dysfunction (74\%). The mean PMOD score increased significantly with increase in number of organ dysfunctions. Also, there was a positive correlation between PMOD scores with number of organs dysfunction $\left(\mathrm{r}=-0.542 ; \mathrm{R}^{2}=0.294 ; \mathrm{p}<\right.$ 0.001), which confirms the strong relationship between PMOD scores and number of organ dysfunction.

Studies to develop and validate PMOD score, both in children and adults have reported similar association of increase in PMOD scores with increase in organ dysfunction. The Indian studies ${ }^{22}$ validating scoring system to predict outcome have also reported similar observation of increase in the predictive scores with increase in number of organ dysfunction. These observations in our and other studies demonstrate that the number of dysfunctional organs can be used to describe severity of cases of MODS, thereby substantiating the use of PMODS as a predictive risk model to evaluate the outcome. However a study by Graciano et al ${ }^{15}$ for the development and validation of PMOD score showed that 4 organ involvement did not predict the outcome of PICU. 
The PMOD score in the study had no significant correlation with the length of hospital stay. The mean PMOD scores were comparable in children with different intervals pertaining to length of hospital stay $(p=0.138)$. A weak negative correlation was observed between PMOD scores and hospital stay $\left(\mathrm{r}=-0.191 ; \mathrm{R}^{2}=0.0365 ; \mathrm{p}=0.056\right)$. A study on MODS association with outcome and mortality by Typpo et $\mathrm{al}^{23}$ (2009), demonstrated that the presence of MODS on the first day of hospitalisation was related to highest mortality and prolonged length of hospital stay. William et $\mathrm{al}^{24}$ (2010) in his study in adult ICU reported that, the duration of hospital stay is not an independent factor and so the correlation of length of hospital stay is not related to outcome of survivor.

Since in this study PMODS have been evaluated as a predictor of outcome, a logistic regression analysis was done on the discharge status (survivors and non-survivors) taking PMODS as a predictor of mortality. It was observed that, log odds of children dying increased by 3.118 unit increase in PMOD score. The odds ratio of this logistic model was 22.591 (95\%, CI 0.56 to $0.83 ; \mathrm{p}<0.001$ ), which states that for an increase of PMOD score by 1 , a child's odds of death increases by $22 \%$. Prediction of probability of death in our PICU according to PMODS showed that a child with PMOD score of 0 had $1.2 \%$ chance of dying in the ICU, whereas a score of 11 predicted $51 \%$ chance of death. This means that the probability of death increases with increase in P-MOD scores. The predicting and observed results were compared by a classification table of goodness and fit model. It was observed that $85 \%$ of the subjects were correctly classified by this model.

To determine the predicting outcome using PMOD score, a receiver Operating Curve was plotted which showed a maximum sensitivity and specificity at a cut-off point of 7.5 (AUC $=0.860 ; 95 \%$ CI $0.761-0.960 ; \mathrm{p}<0.001$ ). This observation confirms PMOD score to be a good predictor of outcome. To determine the predicting outcome using PMOD score, a Receiver Operating Curve was plotted which showed a maximum sensitivity and specificity at a cut-off point of 7.5 (AUC $=0.860 ; 95 \%$ CI $0.761-0.960 ; p<0.001$ ). This observation confirms PMOD score to be a good predictor of outcome. The sensitivity, specificity, PPV and NPV of PMOD scores in predicting mortality was high $(77.78 \%, 82.93 \%$, $50 \%$ and $94.44 \%$ respectively). If PMOD score is $>7.5$ its prediction of non-survival/death is only $50 \%$, whereas if < 7.5 it has a good prediction of survival, i.e. $94 \%$. These findings not only show strong relationship between PMOD scores and outcome, but also predict that higher PMOD scores are significantly associated with mortality.

In a study ${ }^{15}$ for the development and calibration of PMOD score in children, the ROC curve showed 0.92 AUC, which was in comparison with PELOD score by same study. They noted PELOD score more discriminate than the PMOD in predicting the outcome. PMODS was a measure of outcome rather than predictor of outcome in the PICU. Therefore, they concluded that PMODS is a poor predictor of the overall mortality. However, Marshall et $\mathrm{al}^{25}$ reported that predictive power of MOD score in their study measured at the time of admission was better than the power of other scoring system like APACHE II.

One of the drawback of applying PMOD score is not to include Central Nervous System; however, other scoring systems have included descriptors for neurologic dysfunction and have shown that neurologic dysfunction at admission have also role in predicting outcome. The PMODS does not include neurologic dysfunction as the authors reported some of the limitations during the development of scoring system, that is first is observer variability and second is that organ dysfunction evolves during the ICU stay and patients who have a normal GCS, for example at the time of intubation can progress to severe dysfunction during the ICU stay. Hence, efforts to incorporate the neurological dysfunction in the current PMODS system may improve the predictability of the scoring system further.

The limitation of our study is small sample size and we have taken sick patients admitted in ICU and a single centre study, so the recommendation will be to take large sample size and multicentre study.

\section{CONCLUSION}

To conclude from the study that PMOD score strongly correlated with the PICU mortality and number of organ dysfunction and therefore can be used as an effective tool to measure and predict the outcome of the patients admitted to Paediatric Intensive Care Unit by using PMOD.

\section{REFERENCES}

[1] Martha VF, Garcia PC, Piva JP, et al. Comparison of two prognostic scores (PRISM and PIM) at a pediatric intensive care unit. J Pediatr (Rio J) 2005;81(3): 259-64.

[2] Fiser DH. Outcome analysis. In: Rogers MC, Nichols DG, (edr). Textbook of pediatric intensive care. $3^{\text {rd }}$ edn. Baltimore, USA: Williams \& Wilkins 1996:1663-9.

[3] Tan GH, Tan TH, Goh DY, et al. Risk factors for predicting mortality in a paediatric intensive care unit. Ann Acad Med Singapore 1998;27(6):813-8.

[4] Gemke RJ, Bonsel GJ, Vught JVA. Effectiveness and efficiency of a Dutch pediatriac intensive care unit: validity and application of the pediatric risk of mortality score. Critical Care Med 1994;22(9): 1477-84.

[5] Pollack MM, Ruttimann UE, Glass NL, et al. Monitoring patients in pediatric intensive care. Pediatric 1985;76(5):719-24.

[6] Pollack MM, Cuerdon TT, Patel KM, et al. Impact of quality-of-care factors on pediatric intensive care unit mortality. JAMA 1994;272(12):941-6.

[7] Seneff M, Knaus WA, Seneff M. Predicting patient outcome from intensive care: a guide to APACHE, MPM, SAPS, PRISM, and other prognostic scoring systems. J Intensive Care Med 1990;5(1):33-52.

[8] Bertolini G, Donata RAC, Apolone G. PRISM- An assessment of its performance in a sample of 26 italian ICU'S. Crit Care Med 1988;26:1427-32.

[9] Pollack MM, Patel KM, Ruttimann UE. PRISM III: an updated pediatric risk of mortality score. Crit Care Med 1996;24(5):743-52.

[10] Shann F, Pearson G, Slater A, et al. Paediatric index of mortality (PIM): a mortality prediction model for children in intensive care. Intensive Care Med 1997;23(2):201-7. 
[11] Slater A, Shann F, Pearson G. PIM2: a revised version of the paediatric index of mortality. Intensive Care Med 2003;29(2):278-85.

[12] Gunning K, Rowan K. ABC of intensive care: outcome data and scoring systems. BMJ 1999;319(7204):241-4.

[13] Leteurtre S, Martinot A, Duhamel A, et al. Development of a pediatric multiple organ dysfunction score: use of two strategies. Med Decis Making 1999;19(4): 399-410.

[14] Graciano AL, Balko JA, Rahn DS, et al. The pediatric multiple organ dysfunction score (P-MODS): development and validation of an objective scale to measure the severity of multiple organ dysfunction in critically ill children. Crit Care Med 2005;33(7): 1484-91.

[15] Zweig MH, Campbell G. Receiver-operating characteristic: (ROC) plots: a fundamental evaluation tool in clinical medicine. Clin Med 1993;39(4):561-77.

[16] Gandhi J, Sangareddi S, Varadarajan P, et al. Pediatric index of mortality 2 score as an outcome predictor in pediatric intensive care unit in India. Indian J Crit Care Med 2013;17(5):288-91.

[17] Costa GA, Delgado AF, Ferraro A, et al. Application of the pediatric risk of mortality score (PRISM) score and determination of mortality risk factors in a tertiary pediatric intensive care unit. Clinics (Sao Paulo) 2010;65(11):1087-92.

[18] Khilnani P, Sarma D, Singh R, et al. Demographic profile and outcome analysis of a tertiary level pediatric intensive care unit. Indian $\mathrm{J}$ Pediatr 2004;71(7):587-91.
[19] Choi KMS, Ng DKK, Wong SF, et al. Assessment of the pediatric index of mortality (PIM) and the pediatric risk of mortality (PRISM) III score for prediction of mortality in a paediatric intensive care unit in Hong Kong. Hong Kong Med J 2005;11(2):97-103.

[20] Bellad R, Rao S, Patil VD, et al. Outcome of intensive care unit patients using pediatric risk of mortality (PRISM) score. Indian Pediatr 2009;46(12):1091-2.

[21] Kapil D, Bagga A. The profile and outcome of patients admitted to a paediatric intensive care unit. Indan J Paediatr 1993;60(1):5-10.

[22] Shukla VV, Nimbalkar SM, Phatak AG, et al. Critical analysis of PIM2 score applicability in a tertiary care PICU in Western India. International J Pediatr Article ID 703942 2014;2014:7.

[23] Typpo KV, Petersen NJ, Hallman DM, et al. Day 1 multiple organ dysfunction syndrome is associated with poor functional outcome and mortality in the pediatric intensive care unit. Pediatr Crit Care Med 2009;10(5):562-70.

[24] Williams TA, Ho KM, Dobb GJ, et al. Effect of length of stay in intensive care unit on hospital and long-term mortality of critically ill adult patients. Br J Anaesth 2010;104(4):459-64.

[25] Marshall JC, Cook DJ, Christou NV, et al. Multiple organ dysfunction score: a reliable descriptor of a complex clinical outcome. Crit Care Med 1995;23(10):1638-52. 